\title{
Research Article \\ On New Proximal Point Methods for Solving the Variational Inequalities
}

\author{
Muhammad Aslam Noor, ${ }^{1,2}$ Khalida Inayat Noor, ${ }^{1}$ \\ and Eisa Al-Said² \\ ${ }^{1}$ Mathematics Department, COMSATS Institute of Information Technology, Park Road, \\ Islamabad, Pakistan \\ ${ }^{2}$ Mathematics Department, College of Science, King Saud University, Riyadh, Saudi Arabia \\ Correspondence should be addressed to Muhammad Aslam Noor, noormaslam@hotmail.com
}

Received 3 October 2011; Accepted 19 October 2011

Academic Editor: Yonghong Yao

Copyright (C) 2012 Muhammad Aslam Noor et al. This is an open access article distributed under the Creative Commons Attribution License, which permits unrestricted use, distribution, and reproduction in any medium, provided the original work is properly cited.

It is well known that the variational inequalities are equivalent to the fixed point problem. We use this alternative equivalent formulation to suggest and analyze some new proximal point methods for solving the variational inequalities. These new methods include the explicit, the implicit, and the extragradient methods as special cases. The convergence analysis of the new methods is considered under some suitable conditions. Results proved in this paper may stimulate further research in this direction.

\section{Introduction}

Variational inequalities, the origin of which can be traced back to Stampacchia [1], are being used to study a wide class of diverse unrelated problems arising in various branches of pure and applied sciences in a unified framework. It is well known that the variational inequalities are equivalent to the fixed point problem. This alternative equivalent formulation has played an important and fundamental role in the existence, numerical methods, and other aspects of the variational inequalities. This equivalent formulation has been used to suggest the projection iterative method, the implicit iterative method, and the extragradient method, which is due to Korpelevich [2], for solving the variational inequalities. It has been shown [3] that the implicit iterative method and the extragradient method are equivalent. We remark that the implicit iterative method and the explicit iterative method are two different and distinct methods. We use this alternative equivalent formulation to suggest and analyze some new proximal point methods, which include the implicit and explicit methods as special cases. This is the main motivation of this paper. We also consider its convergence criteria 
under suitable conditions. We hope that the ideas and techniques of this paper may stimulate further research in this area of pure and applied sciences.

\section{Preliminaries}

Let $H$ be a real Hilbert space, whose inner product and norm are denoted by $\langle\cdot, \cdot\rangle$ and $\|\cdot\|$, respectively. Let $K$ be a nonempty, closed, and convex set in $H$. such that

For a given nonlinear operator $T: H \rightarrow H$, we consider the problem of finding $u \in K$

$$
\langle T u, v-u\rangle \geq 0, \quad \forall v \in K
$$

which is called the variational inequality, introduced and studied by Stampacchia [1].

For the applications, formulations, numerical methods, and other aspects of the equilibrium variational inequalities, see [1-14] and the references therein.

We now recall some well-known results and concepts.

Lemma 2.1. Let $K$ be a nonempty, closed, and convex set in $H$. Then, for a given $z \in H, u \in K$ satisfies the inequality

$$
\langle u-z, v-u\rangle \geq 0, \quad \forall v \in K,
$$

if and only if

$$
u=P_{K} z
$$

where $P_{K}$ is the projection of $H$ onto the closed and convex set $K$.

It is well known that the projection operator $P_{K}$ is nonexpansive, that is,

$$
\|T u-T v\| \leq\|u-v\|, \quad \forall u, v \in H .
$$

This property plays a very important part in the studies of the variational inequalities and related optimization.

Using Lemma 2.1, one can easily show that the variational inequality (2.1) is equivalent to finding $u \in K$ such that

$$
u=P_{K}[u-\rho T u]
$$

where $\rho>0$ is a constant.

Definition 2.2. An operator $T: H \rightarrow H$ is said to be strongly monotone if and only if there exists a constant $\alpha>0$ such that

$$
\langle T u-T v, u-v\rangle \geq \alpha\|u-v\|^{2}, \quad \forall u, v \in H,
$$


and Lipschitz continuous if there exists a constant $\beta>0$ such that

$$
\|T u-T v\| \leq \beta\|u-v\|, \quad \forall u, v \in H .
$$

\section{Main Results}

In this section, we use the fixed point formulation (2.5) to suggest a new unified implicit method for solving the variational inequality (2.1), and this is the main motivation of this paper. Using the equivalent fixed point formulation, one can suggest the following iterative method for solving the variational inequality (2.1).

Algorithm 3.1. For a given $u_{0} \in K$, find the approximate solution $u_{n+1}$ by the iterative scheme

$$
u_{n+1}=P_{K}\left[u_{n}-\rho T u_{n}\right], \quad n=0,1,2, \ldots
$$

Algorithm 3.1 is known as the projection iterative method. For the convergence analysis of Algorithm 3.1, see Noor [8].

For a given $\lambda \in[0,1]$, we can rewrite (2.5) as

$$
u=P_{K}[u-\rho T u+\lambda \rho(T u-T u)]
$$

This fixed point formulation is used to suggest the following new proximal point iterative method for solving the variational inequality (2.1).

Algorithm 3.2. For a given $u_{0} \in K$, find the approximate solution $u_{n+1}$ by the iterative scheme

$$
u_{n+1}=P_{K}\left[u_{n}-\rho T u_{n+1}+\lambda \rho\left(T u_{n+1}-T u_{n}\right)\right], \quad n=0,1,2, \ldots
$$

Note that Algorithm 3.2 is an implicit-type iterative method. It is clear that for $\lambda=1$, Algorithm 3.2 reduces to Algorithm 3.1. For $\lambda=0$, Algorithm 3.2 collapses to the following implicit iterative method for solving the variational inequality (2.1).

Algorithm 3.3. For a given $u_{0} \in K$, find the approximate solution $u_{n+1}$ by the iterative scheme

$$
u_{n+1}=P_{K}\left[u_{n}-\rho T u_{n+1}\right], \quad n=0,1,2, \ldots
$$

For the convergence analysis of Algorithm 3.3, see Noor [3] and the references therein.

In order to implement Algorithm 3.2, we use the predictor-corrector technique. We use Algorithm 3.1 as the predictor and Algorithm 3.2 as the corrector. Consequently, we obtain the following two-step iterative method for solving the variational inequality (2.1). 
Algorithm 3.4. For a given $u_{0} \in K$, find the approximate solution $u_{n+1}$ by the iterative schemes:

$$
\begin{gathered}
y_{n}=P_{K}\left[u_{n}-\rho T u_{n}\right], \\
u_{n+1}=P_{K}\left[u_{n}-\rho T y_{n}+\lambda \rho\left(T y_{n}-T u_{n}\right)\right], \quad n=0,1,2, \ldots
\end{gathered}
$$

Algorithm 3.4 is a new two-step iterative method for solving the variational inequality (2.1).

For $\lambda=0$, Algorithm 3.4 reduces to the following iterative method for solving the variational inequality (2.1).

Algorithm 3.5. For a given $u_{0} \in K$, find the approximate solution $u_{n+1}$ by the iterative schemes:

$$
\begin{gathered}
y_{n}=P_{K}\left[u_{n}-\rho T u_{n}\right], \\
u_{n+1}=P_{K}\left[u_{n}-\rho T y_{n}\right], \quad n=0,1,2, \ldots
\end{gathered}
$$

which is known as the extragradient method and is due to Korpelevich [2].

For $\lambda=1 / 2$, Algorithm 3.4 reduces to the following iterative method for solving the variational inequality (2.1) and appears to be a new one.

Algorithm 3.6. For a given $u_{0} \in K$, find the approximate solution $u_{n+1}$ by the iterative schemes:

$$
\begin{gathered}
y_{n}=P_{K}\left[u_{n}-\rho T u_{n}\right], \\
u_{n+1}=P_{K}\left[u_{n}-\rho \frac{T y_{n}+T u_{n}}{2}\right], \quad n=0,1,2, \ldots
\end{gathered}
$$

We would like to mention that one can deduce several iterative methods for solving the variational inequality and related optimization problems by choosing the appropriate and suitable value of the parameter $\lambda$. This clearly shows that Algorithm 3.4 is a unified implicit method and includes the previously known implicit and predictor-corrector methods as special cases.

We now consider the convergence criteria of Algorithm 3.4, and this is the main motivation of our next result.

Theorem 3.7. Let the operator $T$ be strongly monotone with constant $\alpha>0$ and Lipschitz continuous with constant $\beta>0$. If there exists a constant $\rho>0$ such that

$$
\theta_{1}=\left[\sqrt{1-2 \lambda \beta \rho+\lambda^{2} \beta^{2} \rho^{2}}+\rho(1-\lambda) \sqrt{1-2 \alpha \rho+\beta^{2} \rho^{2}}\right]<1,
$$

then the approximate solution $u_{n+1}$ obtained from Algorithm 3.4 converges strongly to the exact solution $u \in K$ satisfying the variational inequality (2.1). 
Proof. Let $u \in K$ be a solution of (2.1), and let $u_{n+1}$ be the approximate solution obtained from Algorithm 3.3. Then, from (2.5) and (3.5), we have

$$
\begin{aligned}
\left\|y_{n}-u\right\| & =\left\|P_{K}\left[u_{n}-\rho T u_{n}\right]-P_{K}[u-\rho T u]\right\| \\
& \leq\left\|u_{n}-u-\rho\left(T u_{n}-T u\right)\right\| .
\end{aligned}
$$

From the strongly monotonicity and the Lipschitz continuity of the operator $T$, we obtain

$$
\begin{aligned}
\left\|u_{n}-u-\rho\left(T u_{n}-T u\right)\right\|^{2} & =\left\langle u_{n}-u-\rho\left(T u_{n}-T u\right), u_{n}-u-\rho\left(T u_{n}-T u\right)\right\rangle \\
& =\left\|u_{n}-u\right\|^{2}-\rho\left\langle T u_{n}-T u, u_{n}-u\right\rangle+\rho^{2}\left\|T u_{n}-T u\right\|^{2} \\
& \leq\left(1-2 \alpha \rho+\beta^{2} \rho^{2}\right)\left\|u_{n}-u\right\|^{2}
\end{aligned}
$$

From (3.10) and (3.11), we obtain

$$
\begin{aligned}
\left\|y_{n}-u\right\| & \leq \sqrt{1-2 \alpha \rho+\beta^{2} \rho^{2}}\left\|u_{n}-u\right\| \\
& =\theta\left\|u_{n}-u\right\|
\end{aligned}
$$

where

$$
\theta=\sqrt{1-2 \alpha \rho+\beta^{2} \rho^{2}}
$$

Form (2.5), (3.6), (3.9), (3.12), and (3.13), we have

$$
\begin{aligned}
\left\|u_{n+1}-u\right\| & =\left\|P_{K}\left[u_{n}-\rho T y_{n}+\lambda \rho\left(T y_{n}-T u_{n}\right)\right]-P_{K}[u-\rho T u]\right\| \\
& \leq\left\|u_{n}-u-\lambda \rho\left(T u_{n}-T u\right)\right\|+\rho(1-\lambda)\left\|T y_{n}-T u\right\| \\
& \leq \sqrt{1-2 \alpha \lambda \rho+\beta^{2} \lambda^{2} \rho^{2}}\left\|u_{n}-u\right\|+\rho(1-\lambda) \beta\left\|y_{n}-u\right\| \\
& =\theta_{1}\left\|u_{n}-u\right\|
\end{aligned}
$$

where

$$
\theta_{1}=\left[\sqrt{1-2 \lambda \beta \rho+\lambda^{2} \beta^{2} \rho^{2}}+\rho(1-\lambda) \sqrt{1-2 \alpha \rho+\beta^{2} \rho^{2}}\right]
$$

From (3.9), it follows that $\theta_{1}<1$. Thus the fixed point problem (2.5) has a unique solution, and consequently the iterative solution $u_{n+1}$ obtained from Algorithm 3.3 converges to $u$, the exact solution of (2.5).

For a given $\lambda \in[0,1]$, we can rewrite (2.5) as

$$
u=P_{K}[u-\rho T\{(1-\lambda) u+\lambda u\}] .
$$


This fixed point formulation (3.16) has been used to suggest and analyze the following unified proximal methods for solving the variational inequality (2.1).

Algorithm 3.8. For a given $u_{0} \in K$, find the approximate solution $u_{n+1}$ by the iterative scheme

$$
u_{n+1}=P_{K}\left[u_{n}-\rho T\left\{(1-\lambda) u_{n+1}+\lambda u_{n}\right\}\right], \quad n=0,1,2, \ldots
$$

For the convergence analysis of Algorithm 3.8, see Noor [10]. For different and appropriate choice of the parameter $\lambda$, Algorithm 3.8 includes the extragradient method of Korpelevich [2] and other methods as special cases.

We would like to mention that if the operator $T$ is linear, then Algorithm 3.4 and Algorithm 3.8 are equivalent. In this case, one can easily prove that the convergence of Algorithm 3.4 requires only the partially relaxed strong monotonicity of the operator $T$, which is a weaker condition.

\section{Conclusion}

In this paper, we have used the equivalence between the variational inequality and the fixed point problem to suggest and analyze some new proximal point methods for solving the variational inequality. We have also shown that these new implicit methods include the extragradient method of Korpelevich [2] and the classical implicit method as special cases. We have also discussed the convergence criteria of the proposed new iterative methods under some suitable conditions. Results proved in this paper may inspire further research in this area. It is an open problem to consider the implementation of these new proximal methods and the comparison with other methods. Using the ideas and techniques of this, one can suggest and analyze several new proximal point methods for solving the general variational inequality and its variant form.

\section{Acknowledgments}

This research is supported by the Visiting Professor Program of King Saud University, Riyadh, Saudi Arabia, and the Research Grant no. KSU.VPP.108. The authors are also grateful to Dr. S. M. Junaid Zaidi, Rector, COMSATS Institute of Information Technology, Pakistan, for providing the excellent research facilities.

\section{References}

[1] G. Stampacchia, "Formes bilineaires coercitives sur les ensembles convexes," Comptes Rendus de L'Académie des Sciences, vol. 258, pp. 4413-4416, 1964.

[2] G. M. Korpelevich, "An extragradient method for finding saddle points and for other problems," Ekonomika i Matematicheskie Metody, vol. 12, no. 4, pp. 747-756, 1976.

[3] M. A. Noor, "On an implicit method for nonconvex variational inequalities," Journal of Optimization Theory and Applications, vol. 147, no. 2, pp. 411-417, 2010.

[4] F. Giannessi and A. Maugeri, Variational Inequalities and Network Equilibrium Problems, Plenum Press, New York, NY, USA, 1995.

[5] F. Giannessi, A. Maugeri, and P. M. Pardalos, Equilibrium Problems: Nonsmooth Optimization and Variational Inequality Methods, Nonconvex Optimization and Its Applications, Kluwer Academic, Dordrecht, The Netherlands, 2001.

[6] R. Glowinski, J. L. Lions, and R. Tremolieres, Numerical Analysis of Variational Inequalities, NorthHolland, Amsterdam, Holland, 1981. 
[7] M. A. Noor, "General variational inequalities," Applied Mathematics Letters, vol. 1, no. 2, pp. 119-121, 1988.

[8] M. A. Noor, "Some developments in general variational inequalities," Applied Mathematics and Computation, vol. 152, no. 1, pp. 199-277, 2004.

[9] M. A. Noor, “Extended general variational inequalities," Applied Mathematics Letters, vol. 22, no. 2, pp. 182-185, 2009.

[10] M. A. Noor, On Unified Proximal Method for Variational Inequalities. In press, COMSATS Institute of Information Technology, Islamabad, Pakistan, 2011.

[11] M. A. Noor, E. Al-Said, K. I. Noor, and Y. Yao, "Extragradient methods for solving nonconvex variational inequalities," Journal of Computational and Applied Mathematics, vol. 235, no. 9, pp. 31043108, 2011.

[12] M. A. Noor, K. I. Noor, S. Zainab, and E. Al-Said, "Proximal algorithms for solving mixed bifunction variational inequalities," International Journal of Physical Sciences, vol. 6, no. 17, pp. 4203-4207, 2011.

[13] M. A. Noor, K. I. Noor, S. Zainab, and E. Al-Said, "Some iterative algorithms for solving regularized mixed quasi variational inequalities," International Journal of Physical Sciences, vol. 6, 2011.

[14] M. A. Noor, K. I. Noor, and T. M. Rassias, "Some aspects of variational inequalities," Journal of Computational and Applied Mathematics, vol. 47, no. 3, pp. 285-312, 1993. 


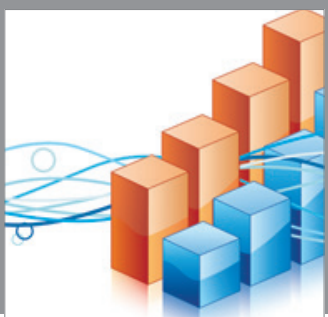

Advances in

Operations Research

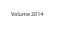

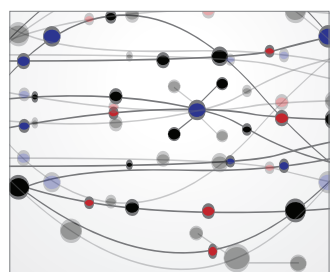

\section{The Scientific} World Journal
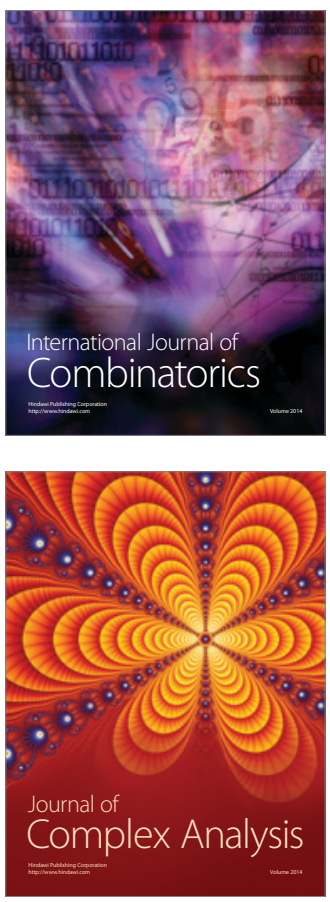

International Journal of

Mathematics and

Mathematical

Sciences
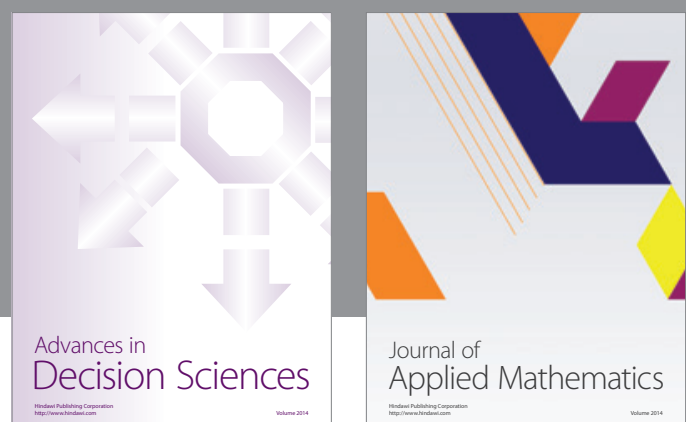

Journal of

Applied Mathematics
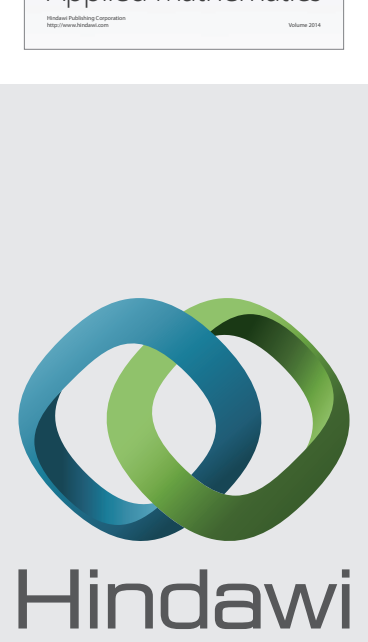

Submit your manuscripts at http://www.hindawi.com
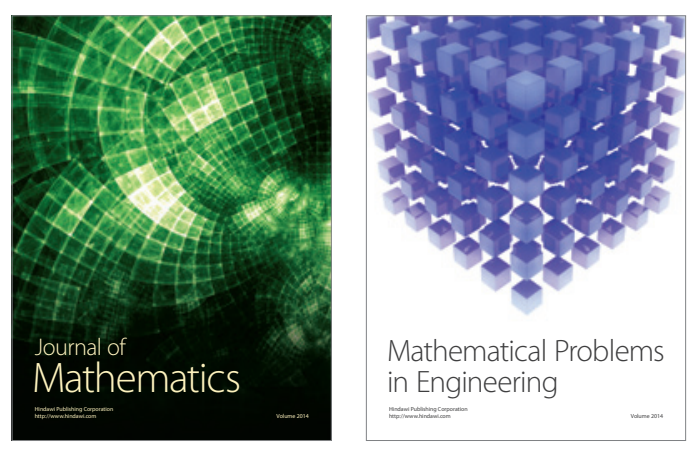

Mathematical Problems in Engineering
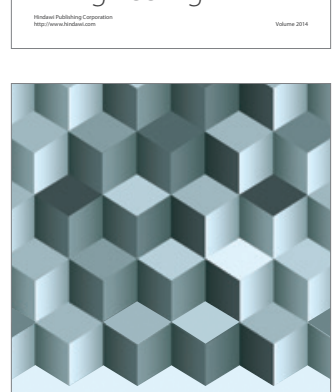

Journal of

Function Spaces
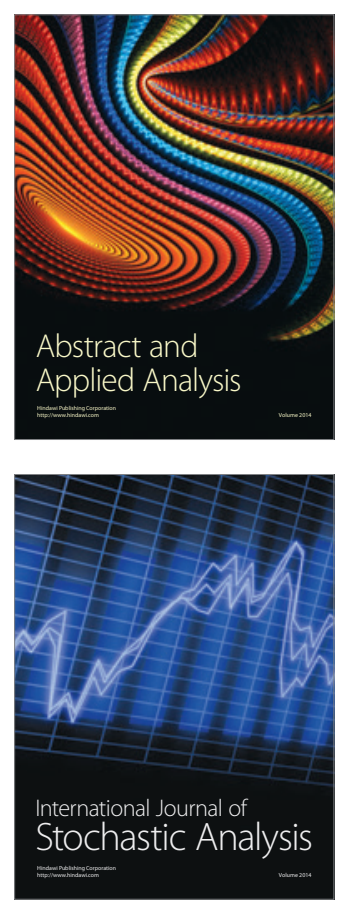

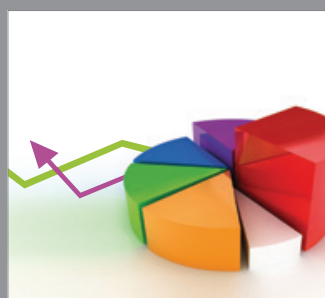

ournal of

Probability and Statistics

Promensencen
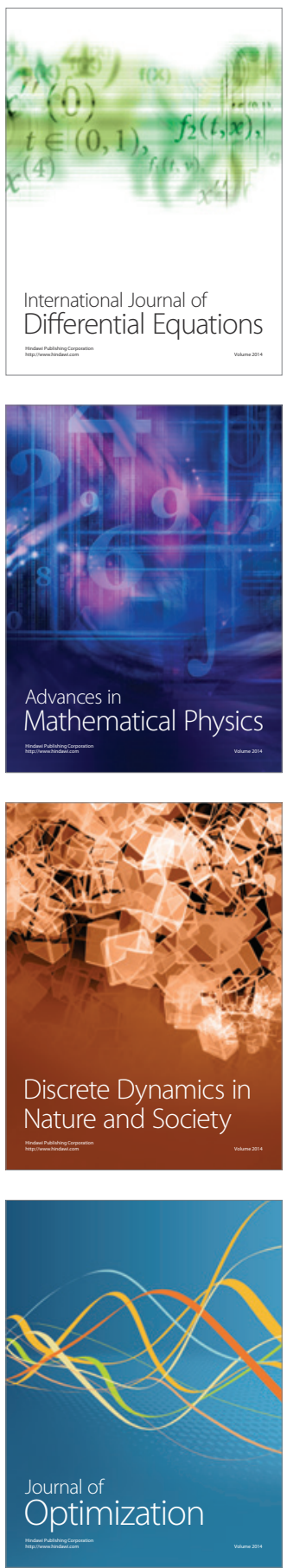